\title{
Year around Flowering Strategy for Jasminum sambac L.
}

\author{
K. R. Zala*, S. T. Bhatt, H. M. Patel, B. M. Tandel, G. D. Patel and Dipal S. Bhatt \\ Department of Floriculture and Landscape Architecture, ACHF, NAU, \\ Navsari, Gujarat, India \\ *Corresponding author
}

\section{A B S T R A C T}

\section{Keywords}

Broad leaf, Mullai, Gundumalli /

Malligai / Arabian jasmine /Tuscan jasmine

Article Info

\section{Accepted:}

20 March 2021

Available Online:

10 April 2021
The present research work entitled 'Year around flowering strategy for Jasminum sambac L' was carried out at Floriculture Research Farm, ASPEE College of Horticulture and Forestry, Navsari Agricultural University, Navsari during 2017-18. It was conducted using various treatments including foliar application of stimulants like $\mathrm{FeSO}_{4}(0.5 \%)$, $\mathrm{ZnSO}_{4}(0.5 \%)$ and Panchgavya (1\%) along with the tip pruning in the month of June. Among the vegetative parameters (viz., plant height and leaf area) there was no significant difference was observed among various treatments. Whereas, in case of the flower quality (viz., flower bud diameter, flower size fully open, flower bud size and weight of 100 flower buds) and yield parameters were significantly influenced by treatment $\mathrm{T}_{7}-$ $\mathrm{FeSO}_{4}(0.5 \%)+\mathrm{ZnSO}_{4}(0.5 \%)$ twice after pruning + tip pruning in June + $\mathrm{FeSO}_{4}(0.5 \%)+\mathrm{ZnSO}_{4}(0.5 \%)$ twice after tip pruning.

\section{Introduction}

Jasmine is a genus of shrubs and vines in the olive family oleaceae. It contains around 200 species native to tropical and warm regions of Eurasia, Australia and Oceania. Jasmines are widely cultivated for the characteristic fragrance of their flowers. Major species of jasmine are Jasminum auriculatum - Mullai, Jasminum grandiflorum - Jathimalli (or) Pitchi (or) Spanish jasmine, Jasminum sambac - Gundumalli / Malligai / Arabian
jasmine/Tuscan jasmine, Jasminum pubescens - Kakada. Jasminum sambac, commonly called Arabian jasmine, is probably native to India or Southeast Asia where it is a broad leaf evergreen shrub. Jasminum sambac produces good yield during the months starting from March to August. The remaining months are considered as off season. Hence, it is important to produce flowers year around to meet out the market demand of flowers. Pruning in jasmine helps to encourage growth of new healthy shoots which bears more 
flowers than old branch. It also helps to maintain its shape and form. Flowering management in jasmine using pruning with application of stimulants is a practice that enables the scheduling of production for periods when the flower value is higher. The practice of tip pruning in productive branches prevents continuous vegetative growth and higher rate of flowering in axillary buds. Due to tip pruning there is reduction in the auxin content of the apices. It momentarily alters the direction of translocation of assimilates, allocating more assimilates towards stimulating the floral emission of the axillary buds of the branches in the flowering conditions and thus the fixation of inflorescence (Oliveira et al., 2016). By introducing the various stimulants and pruning technique, it may be possible to induce round the year flowering and trying to fetch the better price in market.

\section{Materials and Methods}

The present investigation 'Year around flowering strategy for Jasminum sambac L' was carried out at Floriculture Research Farm, ASPEE College of Horticulture and Forestry, Navsari Agricultural University, Navsari during 2017-18. The experiment was laid out in Randomized Blocked Design with three replication and ten treatments viz. $\mathrm{T}_{1}$ Control, $\mathrm{T}_{2}-\mathrm{FeSO}_{4}(0.5 \%)+\mathrm{ZnSO}_{4}(0.5 \%)$ twice after pruning, $\mathrm{T}_{3}$ - Panchgavya (1\%) twice after pruning, $\mathrm{T}_{4}$ - Tip pruning in June, $\mathrm{T}_{5}-\mathrm{FeSO}_{4}(0.5 \%)+\mathrm{ZnSO}_{4}(0.5 \%)$ twice after pruning + tip pruning in June, $\mathrm{T}_{6}$ Panchgavya $1 \%$ twice after pruning + tip pruning in June, $\mathrm{T}_{7}-\mathrm{FeSO}_{4}(0.5 \%)+\mathrm{ZnSO}_{4}$ $(0.5 \%)$ twice after pruning + tip pruning in June $+\mathrm{FeSO}_{4}(0.5 \%)+\mathrm{ZnSO}_{4}(0.5 \%)$ twice after tip pruning, $\mathrm{T}_{8}$ - Panchgavya $1 \%$ twice after pruning + tip pruning in June + Panchgavya $1 \%$ twice after tip pruning, $\mathrm{T}_{9}$ $\mathrm{FeSO}_{4}(0.5 \%)+\mathrm{ZnSO}_{4}(0.5 \%)$ twice after pruning $+\mathrm{FeSO}_{4}(0.5 \%)+\mathrm{ZnSO}_{4}(0.5 \%)$ twice after tip pruning, $\mathrm{T}_{10}$ - Panchgavya $1 \%$ twice after pruning + Panchgavya $1 \%$ twice after tip pruning. The gross plot size of the experiment $4.8 \times 4.8 \mathrm{~m}$ and net plot size was $2.4 \times 2.4 \mathrm{~m}$. The spacing was $1.2 \times 1.2 \mathrm{~m}$. Three four year old plants of Jasminum sambac L. were selected for experiment. Pruning was done at $50 \mathrm{~cm}$ height from the ground level in December, 2017 to get uniform height.

The preparation of solution was done as per the said concentration. First spray was done 7 days after pruning and then $2^{\text {nd }}$ spray was done after 25 days of first spray with the help of knapsack sprayer. Wheras, the tip pruning was done in the first week of June and the third spray was done immediately after tip pruning in June and fourth spray was done after 25 days of third spray. Fresh stimulants solution was prepared at time of each spray and used immediately. Control plants were sprayed with water. All four plants were tagged for recording the observations and average value for each net plot was computed and recorded.

\section{Results and Discussion}

\section{Vegetative parameters}

Effect of different growth stimulants and tip pruning had non significant effect on vegetative growth parameters in jasmine as discussed below. The data showed that effect of various growth stimulants and tip pruning was found non significant regarding plant height and leaf area in jasmine.

\section{Flowering and Yield parameters}

The application of various stimulants and tip pruning had significant effect on flower quality parameters in jasmine from the month of February to December. It is evident from the data that during February, June, July, September and November month, weight of 
100 flower buds significantly influenced due to different treatments of stimulants and tip pruning while, in rest of the months it showed non significant effect. Plants treated with $\mathrm{T}_{7}$ $\left[\mathrm{FeSO}_{4}(0.5 \%)+\mathrm{ZnSO}_{4}(0.5 \%)\right.$ twice after pruning + tip pruning in June $+\mathrm{FeSO}_{4}(0.5 \%)$ $+\mathrm{ZnSO}_{4}(0.5 \%)$ twice after tip pruning] gained maximum weight of 100 flower bud (33.94 g) in June month.

Plants treated with $\mathrm{T}_{7}\left[\mathrm{FeSO}_{4}(0.5 \%)+\mathrm{ZnSO}_{4}\right.$ $(0.5 \%)$ twice after pruning + tip pruning in June $+\mathrm{FeSO}_{4}(0.5 \%)+\mathrm{ZnSO}_{4}(0.5 \%)$ twice after tip pruning] gained maximum flower bud length consistently from Jan to May month. Moreover, flower bud diameter was found consistently higher in the plants treated treatment with $\mathrm{T}_{7}\left[\mathrm{FeSO}_{4}(0.5 \%)+\mathrm{ZnSO}_{4}\right.$ $(0.5 \%)$ twice after pruning + tip pruning in June $+\mathrm{FeSO}_{4}(0.5 \%)+\mathrm{ZnSO}_{4}(0.5 \%)$ twice after tip pruning] in the months of January to May. The increment in flower size might be due to the accelerated mobility of photosynthetic from the source to sink due to the readily available from the panchgavya which contains beneficial microorganisms, growth hormones and vital nutrients for plant growth and quality flower.

The same treatment also resulted significant increase in length of flower bud $(17.80 \mathrm{~mm}$, $17.41 \mathrm{~mm}, 15.98 \mathrm{~mm}, 15.28 \mathrm{~mm}, 16.87 \mathrm{~mm}$, $13.38 \mathrm{~mm}$ and $15.98 \mathrm{~mm}$ ) as well as flower bud diameter $(7.07 \mathrm{~mm}, 6.92 \mathrm{~mm}, 8.25 \mathrm{~mm}$, $8.43 \mathrm{~mm}, 7.66 \mathrm{~mm}, 7.16 \mathrm{~mm}$ and $7.63 \mathrm{~mm}$ ) during June to December months of experimentation. The proliferation in flowering attributes might be due to the beneficial role of zinc and iron. In plants, $\mathrm{Zn}$ is vital for growth, tolerance to stress and chlorophyll synthesis (Kawachi et al., 2009; Lee et al., 2010). It also plays an important role in a wide range of processes, such as growth hormone production and internodes elongation. Iron is a co factor for a large number of enzymes that catalyze several biochemical processes within the plant (Brittenham, 1994; Marschner, 1995). It plays a vital role in the chlorophyll formation, thylakoid synthesis, chloroplast development and functions in the respiratory enzymes. Moreover, iron serves in the transportation of energy in the plant (Miller et al., 1995).

Table.1 Effect of different stimulants and tip pruning on plant height $(\mathrm{cm})$ in Jasminum sambac L.

\begin{tabular}{|c|c|}
\hline Treatments & Plant height $(\mathbf{c m})$ \\
\hline $\mathbf{T}_{\mathbf{1}}$ & 174.83 \\
\hline $\mathbf{T}_{\mathbf{2}}$ & 177.58 \\
\hline $\mathbf{T}_{\mathbf{3}}$ & 176.33 \\
\hline $\mathbf{T}_{\mathbf{4}}$ & 174.91 \\
\hline $\mathbf{T}_{\mathbf{5}}$ & 176.25 \\
\hline $\mathbf{T}_{\mathbf{6}}$ & 177.75 \\
\hline $\mathbf{T}_{\mathbf{7}}$ & 176.50 \\
\hline $\mathbf{T}_{\mathbf{8}}$ & 179.75 \\
\hline $\mathbf{T}_{\mathbf{9}}$ & 176.33 \\
\hline $\mathbf{T}_{\mathbf{1 0}}$ & 177.42 \\
\hline S. Em. $\mathbf{E}$ & 1.63 \\
\hline C. D. at 5 \% & $\mathrm{NS}$ \\
\hline C. $\mathbf{~ V . ~} \%$ & 1.60 \\
\hline
\end{tabular}


Table.2 Effect of different stimulants and tip pruning on leaf area $\left(\mathrm{cm}^{2}\right)$ in Jasminum sambac L.

\begin{tabular}{|c|c|c|c|c|c|c|c|c|c|c|c|c|}
\hline \multirow[t]{2}{*}{ Treatment } & \multicolumn{12}{|c|}{ Leaf Area $\left(\mathrm{cm}^{2}\right)$ (Monthwise) } \\
\hline & Jan & Feb & Mar & Apr & May & Jun & July & Aug & Sept & Oct & Nov & Dec \\
\hline $\mathbf{T}_{1}$ & 21.81 & 20.50 & 20.78 & 23.70 & 26.03 & 21.60 & 21.34 & 22.38 & 23.31 & 24.44 & 25.59 & 26.62 \\
\hline $\mathbf{T}_{2}$ & 22.33 & 23.92 & 21.62 & 25.67 & 26.31 & 22.95 & 22.64 & 23.12 & 24.28 & 24.68 & 26.73 & 27.77 \\
\hline $\mathbf{T}_{\mathbf{3}}$ & 22.65 & 24.02 & 21.68 & 26.42 & 26.66 & 22.96 & 22.92 & 24.12 & 24.30 & 24.80 & 27.70 & 28.48 \\
\hline $\mathbf{T}_{4}$ & 22.10 & 22.91 & 21.15 & 24.95 & 26.21 & 22.21 & 22.43 & 23.10 & 23.86 & 24.61 & 26.26 & 27.14 \\
\hline $\mathbf{T}_{5}$ & 22.94 & 24.26 & 21.70 & 26.79 & 26.77 & 23.58 & 23.51 & 24.92 & 24.54 & 24.86 & 28.06 & 28.82 \\
\hline $\mathbf{T}_{6}$ & 23.51 & 24.28 & 22.04 & 27.06 & 26.78 & 24.47 & 23.73 & 24.95 & 24.60 & 25.00 & 28.06 & 29.52 \\
\hline $\mathbf{T}_{7}$ & 25.04 & 28.08 & 23.24 & 30.47 & 29.60 & 28.87 & 27.31 & 26.70 & 25.69 & 28.07 & 30.99 & 32.09 \\
\hline $\mathbf{T}_{8}$ & 24.49 & 25.67 & 23.13 & 27.87 & 28.53 & 25.60 & 26.91 & 26.55 & 24.94 & 25.40 & 29.64 & 30.93 \\
\hline $\mathbf{T}_{9}$ & 23.90 & 24.33 & 22.18 & 27.06 & 27.56 & 24.74 & 23.81 & 24.98 & 24.74 & 25.20 & 28.07 & 29.75 \\
\hline $\mathbf{T}_{10}$ & 23.97 & 25.39 & 23.08 & 27.39 & 27.89 & 25.51 & 24.52 & 25.55 & 24.77 & 25.39 & 29.01 & 26.62 \\
\hline S.Em. \pm & 1.21 & 0.23 & 1.46 & 1.25 & 1.42 & 1.80 & 1.89 & 1.12 & 1.39 & 1.65 & 1.11 & 1.18 \\
\hline C.D.@ $5 \%$ & NS & NS & NS & NS & NS & NS & NS & NS & NS & NS & NS & NS \\
\hline C.V. \% & 8.99 & 1.42 & 10.23 & 8.10 & 9.01 & 12.88 & 13.69 & 7.87 & 9.84 & 11.33 & 6.88 & 6.98 \\
\hline
\end{tabular}

Table.3 Effect of different stimulants and tip pruning on 100 flower buds wt. (g) in Jasminum sambac L.

\begin{tabular}{|c|c|c|c|c|c|c|c|c|c|c|c|c|}
\hline \multirow[t]{2}{*}{ Treatment } & \multicolumn{12}{|c|}{100 flower buds wt. (g) (Monthwise) } \\
\hline & Feb & Mar & Apr & May & Jun & July & Aug & Sept & Oct & Nov & Dec & Feb \\
\hline $\mathbf{T}_{1}$ & 21.59 & 26.71 & 27.31 & 28.13 & 26.51 & 22.56 & 26.62 & 27.63 & 28.33 & 25.77 & 25.79 & 21.59 \\
\hline $\mathbf{T}_{2}$ & 22.69 & 27.61 & 29.20 & 29.40 & 30.94 & 27.69 & 27.77 & 28.82 & 29.09 & 29.66 & 28.00 & 22.69 \\
\hline $\mathbf{T}_{3}$ & 22.75 & 28.2 & 30.93 & 30.97 & 31.41 & 28.59 & 28.48 & 29.66 & 30.20 & 30.40 & 28.08 & 22.75 \\
\hline $\mathbf{T}_{4}$ & 21.72 & 27.48 & 27.72 & 29.16 & 28.60 & 25.72 & 27.14 & 28.32 & 28.48 & 26.96 & 26.98 & 21.72 \\
\hline $\mathbf{T}_{5}$ & 23.75 & 28.33 & 31.02 & 30.99 & 31.64 & 28.83 & 28.82 & 30.53 & 30.29 & 30.63 & 28.77 & 23.75 \\
\hline $\mathbf{T}_{6}$ & 23.88 & 28.54 & 31.31 & 31.87 & 32.31 & 29.81 & 29.52 & 30.92 & 30.58 & 31.67 & 29.32 & 23.88 \\
\hline $\mathbf{T}_{7}$ & 25.07 & 29.85 & 32.35 & 33.78 & 33.94 & 30.79 & 32.09 & 33.18 & 31.62 & 33.80 & 30.89 & 25.07 \\
\hline $\mathbf{T}_{8}$ & 24.29 & 29.66 & 32.21 & 32.99 & 33.59 & 30.56 & 30.93 & 32.15 & 31.48 & 32.51 & 30.01 & 24.29 \\
\hline $\mathbf{T}_{9}$ & 23.92 & 28.68 & 31.77 & 32.01 & 32.92 & 29.93 & 29.75 & 31.81 & 30.98 & 31.84 & 29.55 & 23.92 \\
\hline $\mathbf{T}_{10}$ & 24.29 & 28.82 & 32.01 & 32.07 & 33.56 & 30.00 & 30.69 & 32.14 & 31.04 & 32.15 & 29.69 & 24.29 \\
\hline S.Em.土 & 1 & 1.43 & 1.31 & 1.25 & 1.14 & 1.45 & 1.18 & 0.73 & 1.19 & 1.66 & 1.52 & 1 \\
\hline C.D. @ $5 \%$ & 2.97 & 4.26 & 3.88 & 3.70 & 3.38 & 4.29 & 3.49 & 2.18 & NS & 4.62 & 4.52 & 2.97 \\
\hline C.V. \% & 7.39 & 8.75 & 7.40 & 6.93 & 6.25 & 8.80 & 6.98 & 4.17 & 6.83 & 8.82 & 9.18 & 7.39 \\
\hline
\end{tabular}


Table.4 Effect of different stimulants and tip pruning on Flower bud diameter (mm) in Jasminum sambac L.

\begin{tabular}{|c|c|c|c|c|c|c|c|c|c|c|c|c|}
\hline \multirow[t]{2}{*}{ Treatment } & \multicolumn{12}{|c|}{ Flower bud diameter (mm) (Monthwise) } \\
\hline & Jan & Feb & Mar & Apr & May & Jun & July & Aug & Sept & Oct & Nov & Dec \\
\hline $\mathbf{T}_{1}$ & 5.01 & 5.33 & 5.47 & 5.32 & 4.81 & 4.62 & 5.86 & 5.88 & 4.61 & 4.80 & 5.25 & 5.01 \\
\hline $\mathbf{T}_{2}$ & 5.88 & 5.79 & 7.14 & 6.13 & 5.24 & 5.14 & 7.21 & 7.10 & 5.81 & 6.39 & 6.59 & 5.88 \\
\hline $\mathbf{T}_{3}$ & 5.90 & 5.82 & 7.38 & 6.43 & 5.26 & 5.30 & 7.28 & 7.36 & 5.97 & 6.40 & 6.66 & 5.90 \\
\hline $\mathbf{T}_{4}$ & 5.78 & 5.73 & 5.75 & 5.49 & 5.05 & 4.85 & 5.95 & 5.92 & 5.39 & 5.59 & 5.33 & 5.78 \\
\hline $\mathbf{T}_{5}$ & 6.08 & 5.86 & 7.39 & 6.82 & 5.37 & 5.40 & 7.48 & 7.64 & 6.04 & 6.48 & 6.86 & 6.08 \\
\hline $\mathbf{T}_{6}$ & 6.09 & 5.93 & 7.62 & 6.85 & 6.12 & 5.67 & 7.57 & 7.69 & 6.24 & 6.64 & 6.95 & 6.09 \\
\hline $\mathbf{T}_{7}$ & 7.84 & 7.36 & 8.76 & 8.00 & 7.07 & 6.92 & 8.25 & 8.43 & 7.66 & 7.16 & 7.63 & 7.84 \\
\hline $\mathbf{T}_{8}$ & 6.53 & 6.22 & 8.29 & 7.23 & 6.18 & 6.11 & 8.20 & 8.40 & 6.63 & 7.11 & 7.59 & 6.53 \\
\hline $\mathbf{T}_{9}$ & 6.11 & 6.01 & 7.66 & 6.89 & 6.12 & 5.76 & 7.67 & 7.86 & 6.44 & 6.78 & 7.06 & 6.11 \\
\hline $\mathbf{T}_{10}$ & 6.51 & 6.03 & 7.75 & 7.02 & 6.16 & 5.80 & 7.70 & 8.09 & 6.51 & 7.04 & 7.09 & 6.51 \\
\hline S.Em. \pm & 0.40 & 0.24 & 0.32 & 0.41 & 0.31 & 0.23 & 0.25 & 0.18 & 0.32 & 0.28 & 0.25 & 0.40 \\
\hline C.D. @ 5 \% & 1.19 & 0.72 & 0.96 & 1.21 & 0.93 & 0.67 & 0.75 & 0.54 & 0.94 & 0.84 & 0.75 & 1.19 \\
\hline C.V. \% & 11.26 & 6.94 & 7.64 & 10.67 & 9.42 & 7.05 & 5.95 & 4.23 & 8.99 & 7.62 & 6.50 & 11.26 \\
\hline
\end{tabular}

Table.5 Effect of different stimulants and tip pruning on Flower Bud length (mm) in Jasminum sambac L.

\begin{tabular}{|c|c|c|c|c|c|c|c|c|c|c|c|c|}
\hline \multirow[t]{2}{*}{ Treatment } & \multicolumn{12}{|c|}{ Flower Bud length (mm) (Monthwise) } \\
\hline & Jan & Feb & Mar & Apr & May & Jun & July & Aug & Sept & Oct & Nov & Dec \\
\hline $\mathbf{T}_{1}$ & 10.75 & 10.72 & 13.34 & 13.36 & 12.91 & 12.69 & 10.55 & 10.71 & 12.51 & 9.74 & 11.09 & 10.75 \\
\hline $\mathbf{T}_{2}$ & 12.83 & 11.67 & 14.21 & 15.21 & 15.53 & 14.32 & 12.57 & 12.18 & 14.12 & 11.92 & 13.71 & 12.83 \\
\hline $\mathbf{T}_{3}$ & 12.97 & 11.84 & 14.61 & 15.21 & 15.73 & 14.63 & 12.70 & 12.30 & 14.36 & 12.05 & 13.91 & 12.97 \\
\hline $\mathbf{T}_{4}$ & 11.86 & 11.36 & 14.08 & 14.97 & 15.19 & 14.30 & 12.47 & 10.94 & 14.11 & 11.80 & 13.37 & 11.86 \\
\hline $\mathbf{T}_{5}$ & 13.03 & 11.88 & 14.86 & 16.21 & 15.87 & 14.64 & 12.85 & 12.36 & 14.46 & 12.75 & 14.05 & 13.03 \\
\hline $\mathbf{T}_{6}$ & 13.05 & 11.93 & 15.03 & 16.36 & 15.92 & 14.84 & 13.11 & 12.58 & 15.36 & 12.79 & 14.11 & 13.05 \\
\hline $\mathbf{T}_{7}$ & 16.99 & 16.33 & 17.60 & 17.91 & 17.80 & 17.41 & 15.98 & 15.28 & 16.87 & 13.38 & 15.98 & 16.99 \\
\hline $\mathbf{T}_{8}$ & 13.54 & 12.11 & 16.11 & 16.53 & 16.64 & 15.48 & 13.40 & 14.07 & 16.72 & 13.25 & 14.82 & 13.54 \\
\hline $\mathbf{T}_{9}$ & 13.32 & 11.94 & 15.31 & 16.51 & 16.02 & 14.92 & 13.18 & 12.74 & 15.82 & 12.81 & 14.20 & 13.32 \\
\hline $\mathbf{T}_{10}$ & 13.53 & 11.95 & 15.81 & 16.52 & 16.63 & 15.20 & 13.26 & 13.09 & 16.07 & 13.12 & 14.81 & 13.53 \\
\hline S.Em. \pm & 0.98 & 0.63 & 0.65 & 0.61 & 0.54 & 0.55 & 0.83 & 0.56 & 0.57 & 0.51 & 0.54 & 0.98 \\
\hline C.D. @ $5 \%$ & 2.91 & 1.87 & 1.92 & 0.80 & 1.62 & 1.63 & 2.46 & 1.67 & 1.69 & 1.51 & 1.62 & 2.91 \\
\hline C.V. \% & 12.89 & 8.96 & 7.41 & 6.61 & 5.96 & 6.40 & 11.01 & 7.73 & 6.56 & 7.13 & 6.73 & 12.89 \\
\hline
\end{tabular}


Table.6 Effect of different stimulants and tip pruning on size of fully open flower (cm) in Jasminum sambac L.

\begin{tabular}{|c|c|c|c|c|c|c|c|c|c|c|c|c|}
\hline \multirow[t]{2}{*}{ Treatment } & \multicolumn{12}{|c|}{ Flower Size (mm) (Monthwise) } \\
\hline & Jan & Feb & Mar & Apr & May & Jun & July & Aug & Sept & Oct & Nov & Dec \\
\hline $\mathbf{T}_{1}$ & 1.80 & 1.91 & 2.02 & 2.00 & 1.96 & 2.08 & 2.17 & 2.10 & 2.06 & 1.62 & 1.80 & 1.80 \\
\hline $\mathbf{T}_{2}$ & 2.23 & 2.12 & 2.31 & 2.26 & 2.35 & 2.29 & 2.63 & 2.46 & 2.29 & 2.22 & 2.23 & 2.23 \\
\hline $\mathbf{T}_{\mathbf{3}}$ & 2.23 & 2.27 & 2.37 & 2.36 & 2.40 & 2.38 & 2.70 & 2.49 & 2.35 & 2.26 & 2.23 & 2.23 \\
\hline $\mathbf{T}_{4}$ & 2.20 & 2.07 & 2.02 & 2.04 & 2.25 & 2.15 & 2.36 & 2.43 & 2.25 & 2.06 & 2.20 & 2.20 \\
\hline $\mathbf{T}_{5}$ & 2.24 & 2.35 & 2.39 & 2.44 & 2.40 & 2.41 & 2.70 & 2.56 & 2.39 & 2.31 & 2.24 & 2.24 \\
\hline $\mathbf{T}_{6}$ & 2.28 & 2.44 & 2.44 & 2.51 & 2.40 & 2.46 & 2.75 & 2.57 & 2.41 & 2.31 & 2.28 & 2.28 \\
\hline $\mathbf{T}_{7}$ & 2.65 & 2.97 & 2.90 & 2.75 & 2.74 & 2.60 & 3.06 & 2.78 & 2.87 & 2.59 & 2.65 & 2.65 \\
\hline $\mathbf{T}_{8}$ & 2.63 & 2.71 & 2.73 & 2.59 & 2.56 & 2.60 & 2.93 & 2.66 & 2.59 & 2.38 & 2.63 & 2.63 \\
\hline $\mathbf{T}_{9}$ & 2.46 & 2.56 & 2.44 & 2.53 & 2.45 & 2.46 & 2.83 & 2.61 & 2.41 & 2.33 & 2.46 & 2.46 \\
\hline$T_{10}$ & 2.50 & 2.65 & 2.46 & 2.57 & 2.53 & 2.58 & 2.86 & 2.62 & 2.44 & 2.34 & 2.50 & 2.50 \\
\hline S.Em. \pm & 0.14 & 0.13 & 0.14 & 0.09 & 0.12 & 0.11 & 0.16 & 0.10 & 0.13 & 0.14 & 0.14 & 0.14 \\
\hline C.D. @ 5 \% & 0.41 & 0.40 & 0.42 & 0.27 & 0.34 & 0.34 & 0.47 & 0.29 & 0.40 & 0.43 & 0.41 & 0.41 \\
\hline CV \% & 10.20 & 9.6 & 10.25 & 6.59 & 8.31 & 8.23 & 10.26 & 10.65 & 9.67 & 11.07 & 10.20 & 10.20 \\
\hline
\end{tabular}

Table.7 Effect of different stimulants and tip pruning on Flower yield (g) in Jasminum sambac L.

\begin{tabular}{|c|c|c|c|c|c|c|c|c|c|c|c|c|}
\hline \multirow[t]{2}{*}{ Treatment } & \multicolumn{12}{|c|}{ Flower yield (g) (Monthwise) } \\
\hline & Jan & Feb & Mar & Apr & May & Jun & July & Aug & Sept & Oct & Nov & Dec \\
\hline $\mathbf{T}_{1}$ & 10.75 & 576.10 & 1312.30 & 1055.80 & 688.17 & 230.73 & 83.80 & 43.60 & 36.27 & 12.29 & 3.50 & 10.75 \\
\hline $\mathbf{T}_{2}$ & 23.16 & 775.80 & 1548.03 & 921.83 & 307.47 & 254.97 & 76.63 & 55.50 & 39.80 & 20.13 & 10.73 & 23.16 \\
\hline $\mathbf{T}_{\mathbf{3}}$ & 26.64 & 787.50 & 1543.10 & 1094.90 & 254.73 & 281.27 & 78.50 & 72.73 & 49.47 & 31.37 & 16.10 & 26.64 \\
\hline $\mathbf{T}_{4}$ & 18.53 & 666.60 & 1402.37 & 1067.40 & 97.00 & 119.00 & 304.27 & 243.77 & 105.90 & 40.70 & 23.27 & 18.53 \\
\hline $\mathbf{T}_{5}$ & 23.05 & 776.10 & 1568.47 & 903.67 & 101.83 & 174.00 & 329.83 & 246.20 & 202.23 & 69.93 & 28.17 & 23.05 \\
\hline $\mathbf{T}_{6}$ & 27.66 & 781.03 & 1554.82 & 906.15 & 99.83 & 185.87 & 341.10 & 269.67 & 219.37 & 76.00 & 32.17 & 27.66 \\
\hline $\mathbf{T}_{7}$ & 25.66 & 808.83 & 1604.03 & 930.83 & 138.70 & 355.47 & 439.90 & 290.00 & 204.23 & 88.47 & 42.53 & 25.66 \\
\hline $\mathbf{T}_{8}$ & 28.64 & 792.90 & 1524.80 & 921.53 & 114.93 & 334.73 & 409.33 & 271.23 & 180.43 & 70.43 & 34.60 & 28.64 \\
\hline $\mathbf{T}_{9}$ & 25.37 & 788.20 & 1579.23 & 907.37 & 641.30 & 247.73 & 168.87 & 89.10 & 69.83 & 55.63 & 18.33 & 25.37 \\
\hline $\mathbf{T}_{10}$ & 28.54 & 794.37 & 1544.37 & 900.23 & 673.53 & 288.03 & 192.27 & 98.97 & 74.40 & 58.30 & 19.67 & 28.54 \\
\hline S.Em.土 & 0.97 & 40.94 & 57.24 & 58.97 & 42.69 & 21.53 & 17.26 & 12.54 & 6.27 & 3.69 & 1.55 & 0.97 \\
\hline C.D.@ $5 \%$ & 2.89 & 121.64 & 170.08 & 175.22 & 126.83 & 63.96 & 51.29 & 37.25 & 18.64 & 10.96 & 4.60 & 2.89 \\
\hline CV \% & 7.08 & 9.40 & 6.53 & 10.63 & 23.72 & 15.08 & 12.33 & 12.92 & 9.19 & 12.22 & 11.71 & 7.08 \\
\hline
\end{tabular}


Table.8 Effect of different stimulants and tip pruning on Flower yield (kg/ha) in Jasminum sambac L.

\begin{tabular}{|c|c|c|c|c|c|c|c|c|c|c|c|}
\hline Trt. & \multicolumn{9}{|c|}{ Flower yield (kg/ha) (Monthwise) } \\
\hline & Feb & Mar & Apr & May & Jun & July & Aug & Sept & Oct & Nov & Dec \\
\hline $\mathbf{T}_{\mathbf{1}}$ & 18.67 & 1000.2 & 2278.48 & 1833.13 & 1194.8 & 400.61 & 145.49 & 75.7 & 62.96 & 21.34 & 6.076 \\
\hline $\mathbf{T}_{\mathbf{2}}$ & 40.21 & 1346.9 & 2687.77 & 1860.97 & 533.83 & 442.68 & 133.05 & 96.361 & 69.10 & 34.95 & 18.63 \\
\hline $\mathbf{T}_{\mathbf{3}}$ & 46.25 & 1367.2 & 2679.20 & 1901.02 & 442.28 & 488.34 & 136.29 & 126.28 & 85.88 & 54.46 & 27.95 \\
\hline $\mathbf{T}_{\mathbf{4}}$ & 32.16 & 1157.3 & 2434.85 & 1853.27 & 168.41 & 206.61 & 528.28 & 423.23 & 183.86 & 70.66 & 40.39 \\
\hline $\mathbf{T}_{\mathbf{5}}$ & 40.02 & 1347.5 & 2723.25 & 1545.84 & 176.80 & 302.10 & 572.67 & 427.46 & 351.12 & 121.4 & 48.90 \\
\hline $\mathbf{T}_{\mathbf{6}}$ & 48.02 & 1356.0 & 2699.55 & 1573.30 & 173.33 & 322.71 & 592.23 & 468.20 & 380.87 & 131.9 & 55.84 \\
\hline $\mathbf{T}_{\mathbf{7}}$ & 44.55 & 1404.3 & 2785 & 1674.03 & 240.81 & 617.17 & 763.77 & 503.51 & 354.60 & 153.6 & 73.84 \\
\hline $\mathbf{T}_{\mathbf{8}}$ & 49.72 & 1376.6 & 2647.43 & 1600.01 & 199.55 & 581.18 & 710.70 & 470.92 & 313.27 & 122.2 & 60.07 \\
\hline $\mathbf{T}_{\mathbf{9}}$ & 44.04 & 1368.5 & 2741.94 & 1505.96 & 1113.4 & 430.12 & 293.19 & 154.69 & 121.24 & 96.59 & 31.83 \\
\hline $\mathbf{T}_{\mathbf{1 0}}$ & 49.55 & 1379.2 & 2681.40 & 1476.21 & 1169.4 & 500.09 & 333.82 & 171.83 & 129.17 & 101.2 & 34.14 \\
\hline S.Em. $\mathbf{\pm}$ & 0.17 & 71.1 & 99.4 & 112.7 & 74.1 & 37.4 & 30 & 21.8 & 10.89 & 6.40 & 2.69 \\
\hline CD at 5 \% & 5 & 211.2 & 295.3 & 334.9 & 220.2 & 111 & 89.1 & 64.7 & 32.35 & 19.03 & 7.99 \\
\hline CV \% & 7.06 & 9.39 & 6.53 & 11.60 & 23.71 & 15.08 & 12.33 & 12.91 & 9.19 & 12.21 & 11.71 \\
\hline
\end{tabular}

Table.9 Effect of different stimulants and tip pruning on plant height (cm) in Jasminum sambac L.

\begin{tabular}{|c|c|}
\hline Treatments & Plant height at the end $(\mathbf{c m})$ \\
\hline T1 & 174.83 \\
\hline T2 & 177.58 \\
\hline T3 & 176.33 \\
\hline T4 & 174.92 \\
\hline T5 & 176.25 \\
\hline T6 & 177.75 \\
\hline T7 & 176.50 \\
\hline T8 & 179.75 \\
\hline T9 & 176.33 \\
\hline T10 & 177.42 \\
\hline S.Em. & 1.63 \\
\hline CD @ 5 \% & NS \\
\hline CV \% & 1.6 \\
\hline
\end{tabular}


Table.10 Effect of stimulants and tip pruning on economics of Jasminum sambac L.

\begin{tabular}{|c|c|c|c|c|c|}
\hline Treatments & Yield(kg/ha) & Gross income (Rs./ha) & Gross expenditure (Rs./ha) & Net income (Rs./ha) & BCR \\
\hline $\mathbf{T}_{\mathbf{1}}$ & 6547.486 & 523798.8 & 148419 & 375379.8 \\
\hline $\mathbf{T}_{\mathbf{2}}$ & 7455.407 & 596432.5 & 161219 & 435213.5 \\
\hline $\mathbf{T}_{\mathbf{3}}$ & 7164.449 & 573155.9 & 151235 & 421920.9 \\
\hline $\mathbf{T}_{\mathbf{4}}$ & 6898.165 & 551853.2 & 151919 & 399934.1 \\
\hline $\mathbf{T}_{\mathbf{5}}$ & 7771.412 & 621712.9 & 164719 & 456993.9 \\
\hline $\mathbf{T}_{\mathbf{6}}$ & 7629.951 & 610396.1 & 164719 & 455661.0 \\
\hline $\mathbf{T}_{\mathbf{7}}$ & 8394.712 & 671577 & 175519 & 496058.0 & 2.79 \\
\hline $\mathbf{T}_{\mathbf{8}}$ & 8144.038 & 651936.8 & 155551 & 496385.8 & 2.94 \\
\hline $\mathbf{T}_{\mathbf{9}}$ & 8060.434 & 644834.7 & 172019 & 472815.7 \\
\hline $\mathbf{T}_{\mathbf{1 0}}$ & 7809.42 & 624753.6 & 152051 & 472702.5 \\
\hline
\end{tabular}

Plate.1 General view of the Experiment

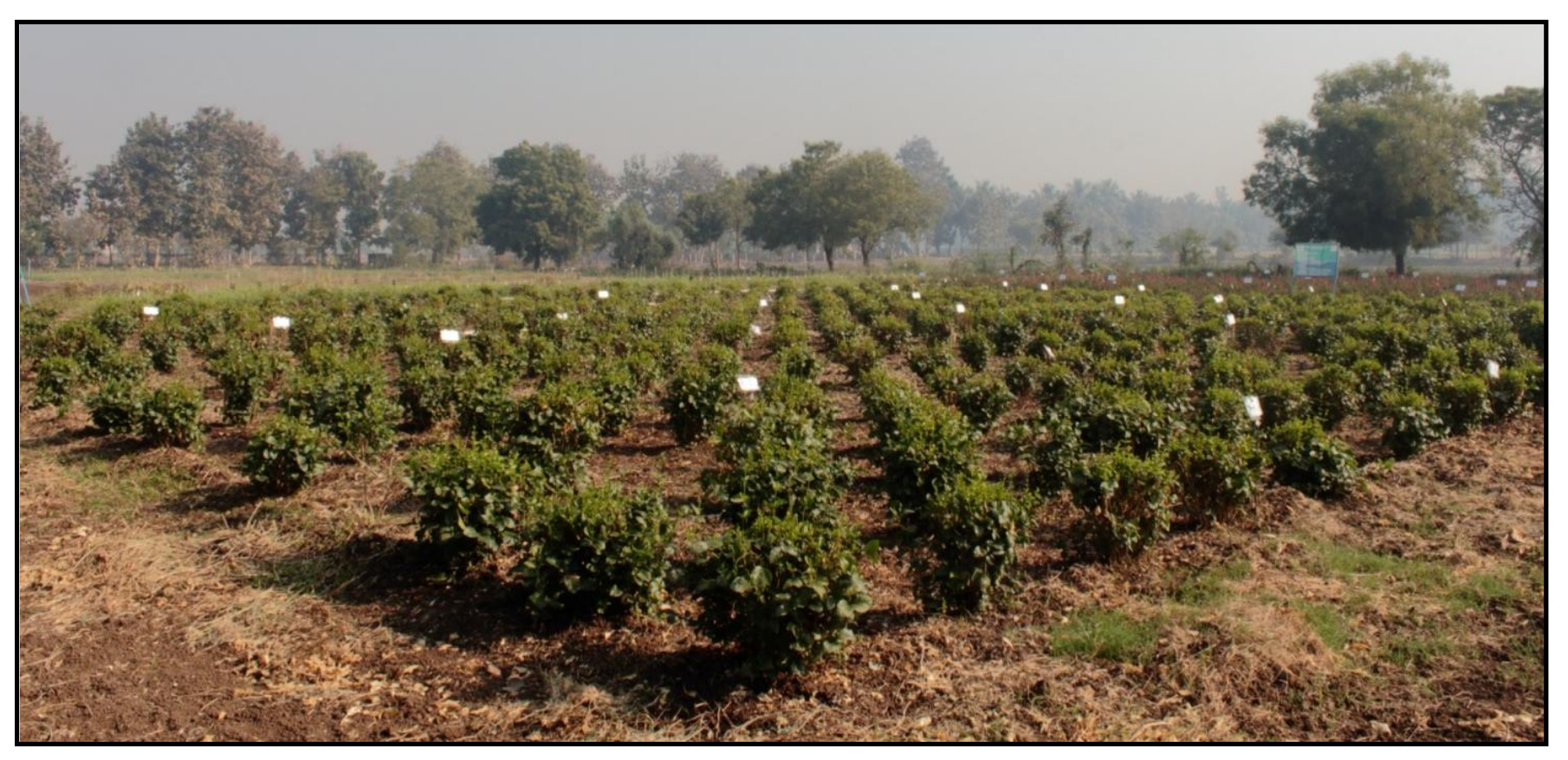


Plate.2 Pruning at $50 \mathrm{~cm}$ height, application of stimulants in and tip pruning at $10 \mathrm{~cm}$ jasmine
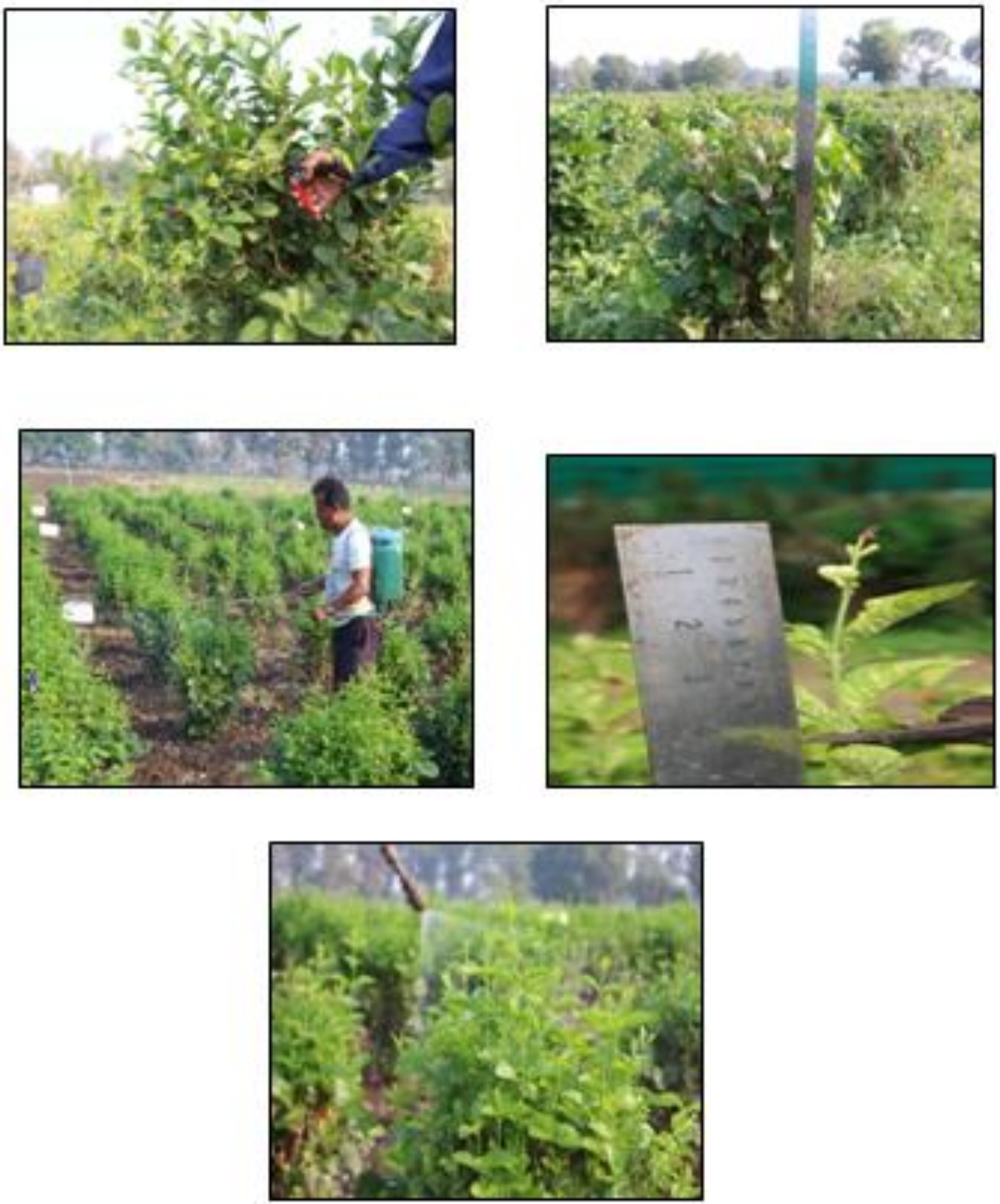


\section{Plate.3}

$\mathrm{T}_{7}-0.5 \% \mathrm{FeSO}_{4}+0.5 \% \mathrm{ZnSO}_{4}$ $\mathbf{T}_{1}$ - Control

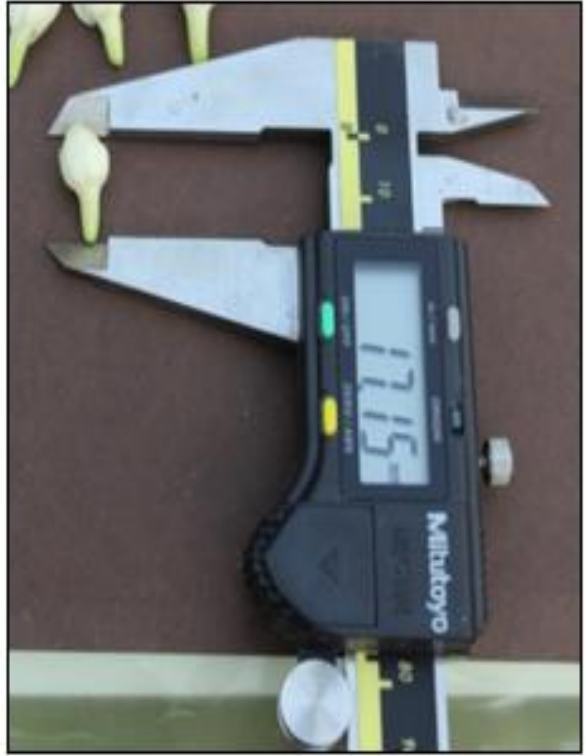

$\mathrm{T}_{7}-0.5 \% \mathrm{FeSO}_{4}+0.5 \% \mathrm{ZnSO}_{4}$

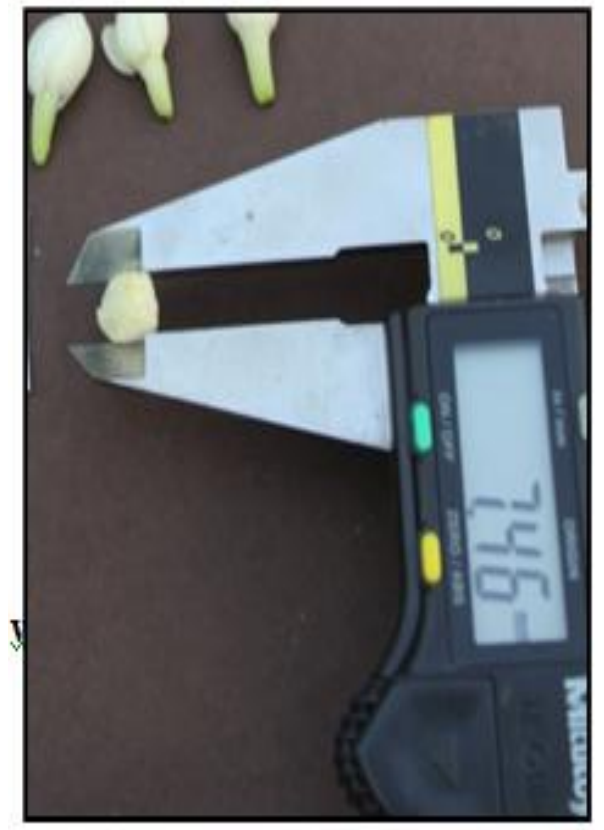

In the months of Jan, Feb, March, April and May largest fully open flower $(2.65 \mathrm{~cm}, 2.97$ $\mathrm{cm}, 2.90 \mathrm{~cm}, 2.75 \mathrm{~cm}$ and $2.74 \mathrm{~cm}$ ) was observed in treatment $\mathrm{T}_{7}\left[\mathrm{FeSO}_{4}(0.5 \%)+\right.$ $\mathrm{ZnSO}_{4}(0.5 \%)$ twice after pruning + tip

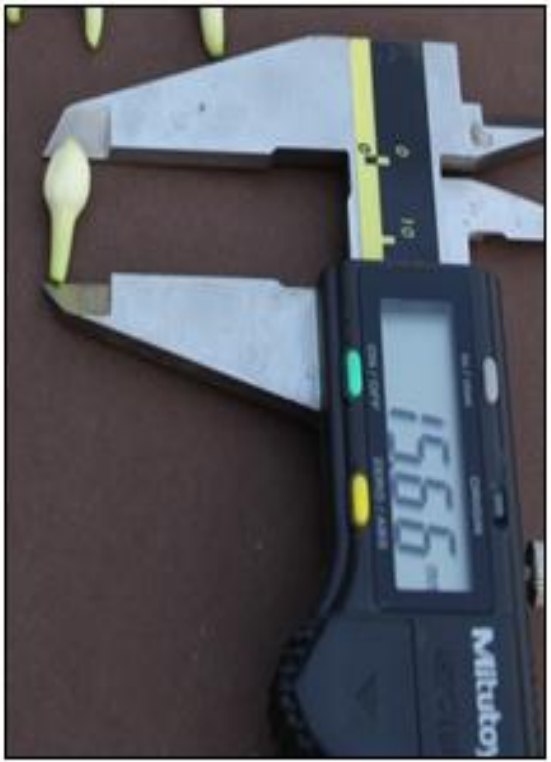

$\mathbf{T}_{1}$ - Control

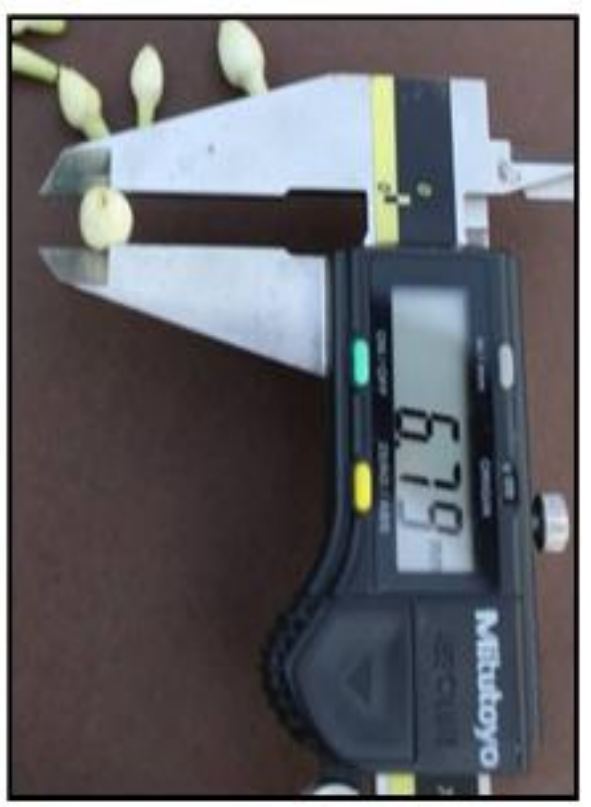

pruning in June $+\mathrm{FeSO}_{4}(0.5 \%)+\mathrm{ZnSO}_{4}$ $(0.5 \%)$ twice after tip pruning]], respectively.

The result is in close conformity with the finding of Sendhilnathan et al., (2017) in 
jasmine. Increase in flower size also might be due to the beneficial role of zinc and iron in enhancing the translocation of carbohydrates, minerals, water and amino acid from the site of synthesis to the storage tissue especially on flowers which increase the size of flowers.

These results are in agreement with the earlier findings of Bhoomi et al., (2018) in jasmine, Karuppaiah (2014) and Neha et al., (2016) in chrysanthemum. During June to December months significantly largest fully open flower $(2.51 \mathrm{~cm}, 2.74 \mathrm{~cm}, 2.60 \mathrm{~cm}, 3.06 \mathrm{~cm}, 2.78 \mathrm{~cm}$, $2.87 \mathrm{~cm}, 2.59 \mathrm{~cm}$ and $2.65 \mathrm{~cm}$, respectively) was obtained from the plants treated with treatment $\mathrm{T}_{7}\left[\mathrm{FeSO}_{4}(0.5 \%)+\mathrm{ZnSO}_{4}(0.5 \%)\right.$ twice after pruning + tip pruning in June + $\mathrm{FeSO}_{4}(0.5 \%)+\mathrm{ZnSO}_{4}(0.5 \%)$ twice after tip pruning].

It is clear from the data that significantly maximum flower bud yield per plot (1604.03 $\mathrm{g}$ and $930.8 \mathrm{~g}$ ) was obtained from the plants treated with treatment $\mathrm{T}_{7}\left[\mathrm{FeSO}_{4}(0.5 \%)+\right.$ $\mathrm{ZnSO}_{4}(0.5 \%)$ twice after pruning] during the month of March and April respectively.

Plants treated with treatment $\mathrm{T}_{7}\left[\mathrm{FeSO}_{4}(0.5 \%)\right.$ $+\mathrm{ZnSO}_{4}(0.5 \%)$ twice after pruning + tip pruning in June $+\mathrm{FeSO}_{4}(0.5 \%)+\mathrm{ZnSO}_{4}$ $(0.5 \%)$ twice after tip pruning] recorded significantly maximum weight of 100 flower buds $(33.94 \mathrm{~g}, 30.79 \mathrm{~g}, 33.18 \mathrm{~g}$ and $33.80 \mathrm{~g})$ in the months of June, July, September and November, respectively. While It might be due to application of zinc and iron not only relieved the chlorosis and produced healthy green plants but also increased the synthesis of chlorophyll, growth promoting substances and mobility of minerals, water, photosynthates and amino acids from the source to sink might have increased flower production and ultimately flower yield. In May month, non significant result was found due to various treatments of stimulants.
During June month, significantly highest flower bud yield per plot (646.94 g) was achieved from plants without any stimulant and pruning treatment $\left(\mathrm{T}_{1}-\mathrm{Control}\right)$. This might be due to the unpruned plants. In the months of July, August and September, significant increase in flower bud yield per plot (355.46 g, 413.23 g and $256.67 \mathrm{~g}$, respectively) was noted in plants treated with treatment $\mathrm{T}_{7}\left[\mathrm{FeSO}_{4}(0.5 \%)+\mathrm{ZnSO}_{4}(0.5 \%)\right.$ twice after pruning + tip pruning in June + $\mathrm{FeSO}_{4}(0.5 \%)+\mathrm{ZnSO}_{4}(0.5 \%)$ twice after tip pruning]. The increase in yield may be due to the micronutrients as iron $(\mathrm{Fe})$ and zinc $(\mathrm{Zn})$ are the trace elements that play essential role in plant growth and increasing crop yields. Zinc favours the storage of more carbohydrates through photosynthesis and iron involves in synthesis of plant hormones and also plays an important role in chlorophyll synthesis, photosynthesis and respiration. This may be the attributing factor for the positive effectiveness of optimum dose of zinc and iron on reducing juvenile phase of the plant. Similar results are also obtained by Bhoomi et al., (2018). Furthermore, the positive response to tip pruning might be due to an optimum balance between the vegetative and reproductive growth of plant. Lightly pruned plants can efficiently supply auxin which has resulted in higher yield in Guava (Prasanna et al., 2018). During October and November, significantly maximum flower bud yield per plot $(219.37 \mathrm{~g}$ and $76.00 \mathrm{~g}$ ) was resulted from plants treated with treatment $\mathrm{T}_{5} \quad \mathrm{FeSO}_{4}$ $(0.5 \%)+\mathrm{ZnSO}_{4}(0.5 \%)$ twice after pruning + tip pruning in June]. Whereas, plants treated with treatment $\mathrm{T}_{7}\left[\mathrm{FeSO}_{4}(0.5 \%)+\mathrm{ZnSO}_{4}\right.$ $(0.5 \%)$ twice after pruning + tip pruning in June $+\mathrm{FeSO}_{4}(0.5 \%)+\mathrm{ZnSO}_{4}(0.5 \%)$ twice after tip pruning] produced highest bud yield per plot $(35.86 \mathrm{~g})$ in December. This enhancement in yield might be due to micronutrients as iron $(\mathrm{Fe})$ and zinc $(\mathrm{Zn})$ are the trace elements that play essential role in plant growth and increasing crop yields. 
Moreover, they improve plant nutrition and increase soil productivity (Marschner, 1995). It is also resulted by Davenport (2006) that the pruning of the tips of branches stimulates the cycles of branching of lateral shoots and removes structures that inhibit reproductive budding originating from the previous productive cycle.

Application of $\mathrm{FeSO}_{4}(0.5 \%)+\mathrm{ZnSO}_{4}(0.5 \%)$ twice after pruning + tip pruning in June + $\mathrm{FeSO}_{4}(0.5 \%)+\mathrm{ZnSO}_{4}(0.5 \%)$ twice after tip pruning $\left(\mathrm{T}_{7}\right)$ to jasmine produced significantly maximum total flower bud yield $(4.83 \mathrm{~kg}$ per plot and $8.39 \mathrm{t}$ per ha).

\section{Economics}

Different investigated stimulants treatment combinations studied in the present investigation were observed to influence profoundly net income and benefit: cost ratio. Maximum benefit: cost ratio (3.19) was obtained from treatment $\mathrm{T}_{8}$ (Panchgavya $1 \%$ twice after pruning + tip pruning in June + Panchgavya 1\% twice after tip pruning).

Based on the findings of present investigation, it can be concluded that foliar application of $\mathrm{FeSO}_{4}(0.5 \%)+\mathrm{ZnSO}_{4}(0.5 \%)$ twice after pruning $\left(1^{\text {st }}\right.$ spray at $7^{\text {th }}$ day after pruning and $2^{\text {nd }}$ spay at $25^{\text {th }}$ day after pruning + tip pruning in June $+\mathrm{FeSO}_{4}(0.5 \%)+\mathrm{ZnSO}_{4}(0.5 \%)$ twice after tip pruning $\left(1^{\text {st }}\right.$ spray immediately after tip pruning and $2^{\text {nd }}$ spray at $25^{\text {th }}$ day after tip pruning) found beneficial to induce year round production of marketable flower buds.

\section{References}

Anonymous (2018).Indian Horticulture Database. National Horticulture Board, Ministry of Agriculture, Government of India, New Delhi.

Anburani, A.; Shakila, A.; and Gayathiri, M. (2008). Effect of organic manures in combination with fertilizers on yield in gundumalli (Jasminum sambac Ait.). Asian J. Hort., 3(2): 419-421.

Bagheri, A.; Ahmad, R.; and Bohloul, A. (2013). The effect of iron chelate foliar application on Damask Rose. Ann. Biol. Res., 4(4): 5355.

Bakshi, P.; Jasrotia, A.; Wali, V. K.; Sharma, A. and Bakshi, M. (2013). Influence of preharvest application of calcium and micronutrients on growth, yield, quality and shelflife of strawberry cv. Chandler. Indian $J$. Agr. Sci., 83(8): 831-835.

Bharti Singh.; Srivastava, R. and Chandra, R. (2007). Response of panchagavya and manchurian mushroom tea on floral characters in Tubrose (Polianthus tuberosa Linn.). J. Ornam. Hort., 10(4): 250-254.

Bhattacharjee, S. K. (1993). Response of micronutrients spray on growth and flowering of rose cv. Raktagandha. Indian Rose Annual., 11: 108-113.

Bhoomi Naik; Bhatt, S. T.; Dipal S. Bhatt.; Dipika Gamit and Patel, M. A. (2018). Economics of off season flower induction through various stimulants in Jasminum sambac L. Biosci. Trends., 1(43).

Brittenham, G. M. (1994). New advances in iron metabolism, iron deficiency, and iron overload. Curr. Opin. Hematol., 1(2): 101106.

Cabrera, R. I.; Evans, R. Y. and Paul, J. L. (1993). Leaching losses of $\mathrm{N}$ from container-grown roses. Sci. Hortic., 53(4): 333-345.

Chakraborty, S.; Sadhukhan, R. and Dey, S. (2009). Integrated nutrient management studies in anthurium. J. Ornam. Hort. 12(4): 265-268.

Neha Chopde.; Borse, G. H.; Ommala, K. and Ghodke, A. T. (2016). Effect of zinc sulphate and ferrous sulphate on growth and flowering of annual chrysanthemum. Plant Archives., 16(2): 594-596.

Davenport, T. L. (2006). Pruning strategies to maximize tropical mango production from the time of planting to restoration of old orchards. Hort. Science., 41(3): 544-548.

Gebremedhin, T. and Rama, A. A. (2015). Efficacy of foliar nutrition on vegetative and reproductive growth of sunflower (Helianthus Annuus L.) 15(9).

Gopu, B.; Balamohan, T. N.; Soman, P. and 
Jeyakumar, P. (2014). Canopy management in mango (Mangifera indica L.) cv. Alphonso with reference to flowering, yield and quality characters under ultra high density planting. J. Appl. Hortic., 16(1).

Halder, N. K.; Ahmed, R.; Sharifuzzaman, S. M.; Anzu-Man Arabagam, K. and Siddiky, M. A. (2007). Effect of boron and zinc fertilization on corm and cormel production of gladiolus in grey terrace soils of Bangladesh. Int. J. Sustain. Crop Prod., 2(5): 85-89.

Hatwar, G. P.; Gondane, S. V.; Urkude, S. M. and Gahukar, O. V. (2003). Effect of micronutrients on growth and yield of chilli. Soil Crop, 13: 123-1254.

Hembrom, R. and Singh, A. K. (2015). Effect of iron and zinc on growth, flowering and bulb yield in lilium. Int. J. Agri. Env. Biotechnology., 8(1): 61-64.

Henaxi, Patel (2016). Effect of foliar application of micronutrients on growth and flowering on rose cv. Top Secret under polyhouse condition. M.Sc. Thesis submitted to Navsari Agricultural University, Navsari.

Jat, R. N.; Khanelwal, S. K. and Gupta, K. N. (2007). Effect of foliar application of urea and zinc sulphate on growth and flowering parameters in african marigold (Tagetes erecta Linn.). J. Ornam. Hort., 10(4): 271273.

Jauhari, S.; Ranjan, S. and Srivastava, P. C. (2005). Effect of zinc on growth, flowering, corm attributes, post-harvest life, leaf and corm nutrient status in gladiolus cv. Red Beauty., Progressive Hort., 37(2): 423.

Kakade, D. K.; Rajpu, G. S. and Joshi, K. I. (2009). Effect of foliar application of ' $\mathrm{Fe}$ ' and ' $\mathrm{Zn}$ ' on growth, flowering and yield of China aster (Callistephus chinensis L. Nees). The Asian J. Hort., 4(1): 138-140.

Karuppaiah, P. (2014). Effect of zinc and iron on growth, yield and quality of chrysanthemum (Dendrathemum grandiflorum Tzeuleu). The Asian J. Hort., 9(1): 232-236.

Kawachi, M.; Kobae, Y.; Mori, H.; Tomioka, R.; Lee, Y. and Maeshima, M. (2009). A mutant strain Arabidopsis thaliana that lacks vacuolar membrane zinc transporter MTP1 revealed the latent tolerance to excessive zinc. Plant Cell Physiol., 50(6): 1156-1170.
Kumar, P. and Arora, J. S. (2000). Effects of micronutrients on gladiolus. J. Ornam. Hort., 3: 91-93.

Kumar, S. and Haripriya, K. (2010). Effect of foliar application of iron and zinc on growth, flowering and yield of nerium (Nerium odoratum L.). Plant Archives., 10(2): 637-640.

Lahijie, M. F. (2012). Application of micronutrients $\mathrm{FeSO}_{4}$ and $\mathrm{ZnSO}_{4}$ on the growth and development of gladiolus variety "Oscar". Intl. J. Agri. Crop Sci., 4(1): 718-720.

Lee, S.; Ryoo, N.; Jeon, J. S.; Guerinot, M. L. and An, G. (2012). Activation of rice Yellow Stripe1-Like 16 (OsYSL16) enhances iron efficiency. Molecules and cells., 33(2): 117126.

Soler, L. and Cuevas, J. (2008). Development of a new technique to produce winter cherimoyas. Hort. Technology., 18(1): 2428.

Marschner, H. (1995). Mineral nutrition of higher plants. $2^{\text {nd }}$. Edn. Academic Pres.

Miller, G. W.; Huang, I. J.; Welkie, G. W. and Pushnik, J. C. (1995). Function of iron in plants with special emphasis on chloroplasts and photosynthetic activity. In Iron nutrition in soils and plants (pp. 19-28). Springer, Dordrecht.

Muthamizhselvi, M. (2006). Integrated nutrient management in chrysanthemum (Dendranthema grandiflora Tzelev.) cv. CO-1. M.Sc. (Hort.) Thesis submitted to Tamil Nadu Agricultural University, Coimbatore, T.N. (INDIA).

Naik, S. K.; Barman, D.; Devadas, R. and Ushabharathi, T. (2013). Evaluation of panchgavya as source of nutrient for Cymbidium orchid. Afr. J. Agric. Res., 8(46): 5728-5732.

Nelson, P.V. (1998). Greenhouse Operation and Management. Practice Hall, Upper Saddle River, NJ.

Oliveira, G. P.; Siqueira, D. L. D.; Salomao, L. C.; Cecon, P. R. and Machado, D. L. M. (2017). Paclobutrazol and branch tip pruning on the flowering induction and quality of mango tree fruits. Pesqui. Agropecu. Trop., 47 (1): 7-14.

Ouellette, D. R. and Young, E. (1994). Branch 
inducement in apple stoolbed shoots by summer leaf removal and tipping. HortScience, 29(12): 1478-1480.

Panse, V. G. and Sukhatme, P. V. (1985). Statistical methods for Agricultural workers. ICAR, New Delhi.

Prasanna, S. G.; Bhagwan, A.; Kumar, K. A. and Saidiah, P (2018). Effects of tip pruning and mulching on fruit development and yield of guava cv. Allahabad safeda, Int. J. Agric. Sci., 8(2): 183-190.

Rawia, A. E.; Khalifa, R. Kh. M. and Shaaban, S. H. A. (2010). Effect of foliar application of zinc and benzyladenine on growth, yield and chemical constituents of tuberose plants. Res. J. Agric. \& Biol. Sci., 6(6): 732743.

Reddy, S. V. G. and Rao, M. B. N. (2012). Precision foliar application of zinc to improve the growth and yield of gladiolus. AIPA, Agri., 344-345.

Saini, T. C.; Polara, N. D. and Bajad, A. V. (2015). Effect of micronutrients ( $\mathrm{Fe}$ and $\mathrm{Zn}$ ) on growth of chrysanthemum (Chrysanthemum morifolium Ramat.). Asian J. Hort., 10(2): 216-221.

Saraswathi, T. and Vadivel, E. (2009). Effect of panchgavya, nitrobenzene and salicylic acid in increasing yield of marigold (Tagetes erecta L.). Advances in Plant Sci., 22(1): 119-121.

Sarkhosh, A.; McConchie, C. and Khadivi, A. (2018). The effects of different tip-pruning times on flowering, yield, and maturity of two mango cultivars in subtropical climate of Northern Territory (Katherine region) from Australia. Sci. Hortic., 234: 140-145.

Schnitzer, M. (2000). A life time perspective on the chemistry of

soil organic matter. Adv. Agron., 68: 3-54.

Sendhilnathan, R.; Velmurugan, V. and Manimaran, P. (2017). Effect of bio regulators along with organics on growth and yield of gundumalli (Jasminum sambac Ait). J. Pharmacogn. Phytochem. 6(5): 234238.

Sharma, B. P.; Gupta, Y. C.; Dhiman, S. R. and Bhalla, R. (2010). Effect of foliar application of biostimulants on growth and flowering of carnation cv. Sunrise. $J$. Ornam. Hort., 13(2): 101-106.

Sivakumar, R.; Chandrasekhar, C. N. and Srividhya, S. (2014). Development of foliar concoction for improving flower yield in jasmine (Jasminum sambac). Asian J. Hort., 9(1): 183-186.

Singh, J. P.; Kumar, K. and Katiyar, P. N. (2012). Effect of zinc, iron and copper on yield parameters of Gladiolus. Hort. Flora. Res. Spectrum., 1(1):64-68.

Sundar, S. B.; Kannan, M. and Jawaharlal, M. (2014). Off Season flower induction through fertigation and biostimulant sprayin Jasminum sambac Ait. Asian J. Hort., 9(1): 32-35.

Thangaselvabai, T.; Jayasekar, M. and K. Eraivan. (2014). Off Season Flower Induction in Jasmine - a Money Spinner. Krishi Vigyan Kendra, Pechiparai, Kanyakumari.

Trivedi, H. and Kumar, A. (2015). Response of bio-enhancers on growth and flowering in rose (Rosa hybrida) cv. Grand Gala. Int. J. Basic Applied Agri. Res., 13(1).

Younis, A.; Riaz, A.; Sajid, M.; Mushtaq, N.; Ahsan, M.; Hameed, M.; Tariq, U. and Nadeem, M. (2013). Foliar application of macro and micronutrients on the yield and quality of Rosa hybrida cvs. Cardinal and Whisky Mac. African Journal of Biotechnology, 12(7): 702-703.

Thangaselvabai, T. Jayasekar, M. and K. Eraivan. 2014. Off Season Flower Induction in Jasmine - a Money Spinner. Krishi Vigyan Kendra, Pechiparai, Kanyakumari.

\section{How to cite this article:}

Zala, K. R., S. T. Bhatt, H. M. Patel, B. M. Tandel, G. D. Patel and Dipal S. Bhatt. 2021. Year around Flowering Strategy for Jasminum sambac L. Int.J.Curr.Microbiol.App.Sci. 10(04): 834847. doi: https://doi.org/10.20546/ijcmas.2021.1004.087 